\title{
Ascertainment of self-reported prescription medication use compared with pharmaceutical claims data
}

\section{Danijela Gnjidic ${ }^{a, h^{*}}$, Wei Du ${ }^{b^{*}}$, Sallie-Anne Pearson ${ }^{\mathrm{a}, \mathrm{c}}$, Sarah N Hilmer ${ }^{\mathrm{d}, \mathrm{e}, \mathrm{f}}$ and Emily Banks b,g, on behalf of the High Risk Prescribing Investigators}

*These authors contributed equally to the work

a Faculty of Pharmacy, University of Sydney, NSW, Australia

${ }^{b}$ National Centre for Epidemiology and Population Health,

Research School of Population Health, Australian National University, Canberra, ACT

${ }^{\circ}$ Centre for Big Data Research in Health, Faculty of Medicine, UNSW Sydney, Australia d Sydney Medical School, University of Sydney, NSW, Australia

e Kolling Institute, Sydney, NSW, Australia

${ }^{\dagger}$ Royal North Shore Hospital, Sydney, NSW, Australia

g Sax Institute, Sydney, NSW, Australia

n Corresponding author: danijela.gnjidic@sydney.edu.au

\section{Article history}

Publication date: October 2017 Citation: Gnjidic D, Du W, Pearson SA, Hilmer SN, Banks E, on behalf of the High Risk Prescribing Investigators. Ascertainment of self-reported prescription medication use compared with pharmaceutical claims data. Public Health Res Pract. 2017;27(4):e27341702. https://doi.org/10.17061/phrp27341702

\section{Key points}

- Overall, self-reported data on the use of prescription-only medications compared well with independent routine data on medication dispensing, with positive predictive values (PPVs) of $\geq 75 \%$ for $79 \%$ of the medications examined

- Sensitivity and PPVs for self-reported versus dispensing data varied markedly by medication type, with excellent validity for long-term chronic prescription medications such as cardiovascular medications, and lower validity for medications used in the short term or intermittently, such as analgesics and antibacterial agents

- Self-reported data on medication use of the type reported here are likely to be useful and valid for classifying exposure to medications, particularly for long-term, regularly used medications

\section{Abstract}

Background: Evidence on the comparative validity of self-reported medication use in large-scale studies is limited. This study compared selfreported medication use of prescription-only medications to gold standard pharmaceutical claims (i.e. dispensing) data.

Methods: We selected a random sample of 500 participants from the 45 and Up Study, a large-scale Australian study, with complete ascertainment of Pharmaceutical Benefits Scheme dispensing records. Self-reported medication use was ascertained by questionnaire requesting data on medications used "for most of the last 4 weeks". In the dispensing data, we determined exposure to specific medications in the same 4-week window as the survey response if we observed a dispensing record $\leq 90$ days before the start of the window. We calculated sensitivity and positive predictive values (PPVs) at the Anatomical Therapeutic Chemical (ATC) classification 3- and 7-digit code levels.

Results: PPVs were $\geq 75 \%$ for $79 \%$ of the medications examined at the 3 -digit ATC level. The sensitivity/PPV of self-reported versus claims data at the 3-digit level were highest for chronic medications, including cardiovascular medications: 94.4\%/96.9\%, respectively, for lipid-lowering agents; $92.5 \% / 97.5 \%$ for angiotensin agents; $88.8 \% / 93.1 \%$ for beta-blockers; and $88.0 \% / 96.9 \%$ for calcium-channel blockers. PPVs were $\geq 65 \%$ and sensitivity of self-reported data was $78.9 \%$ for psychoanaleptics, $42.1 \%$ for analgesics, $26.0 \%$ for psycholeptics and $4.8 \%$ for antibacterial agents. PPVs for individual medications were $\geq 75 \%$ for $81 \%$ of the individual medications examined at the 7-digit level. The sensitivity/PPV for self-reported versus claims data at the 7-digit level varied across individual medications, with highest values being 96.9\%/96.9\% for warfarin, 94.5\%/92.0\% for atorvastatin, 94.3\%/84.6\% for pantoprazole and $93.3 \% / 95.5 \%$ for atenolol. The lowest sensitivity of self-reported versus claims data for individual medications was $16.7 \%$ for temazepam, $15.2 \%$ for perindopril, $11.5 \%$ for irbesartan, $11.1 \%$ for oxazepam and $3.3 \%$ for amoxicillin. 
Conclusions: Self-reported data of the type reported here are useful for identifying exposure to prescription medications, particularly those for chronic use. However, they are likely to be of lesser validity for ascertaining short-term and/or intermittent medication exposure.

\section{Introduction}

High-quality pharmacoepidemiological research relies on accurate ascertainment of medication exposure. The primary ways of ascertaining medication exposure in pharmacoepidemiological studies are using pharmaceutical claims or prescription data, selfreporting, or individual medication inventory at interview. Each has advantages and disadvantages. Claims data are relatively cheap to use, document use over time and are available on a large scale; however, they only document prescribing or dispensing of medications, rather than their actual use, and generally do not include medications that are not subsidised. ${ }^{1}$ Self-report methods can capture information on a wide range of prescription, nonprescription, complementary and alternative medicines reported as being used by the patient. However, there may be issues with accuracy of self-report and recall, and these methods are potentially costly to apply on a large scale. A formal medication review is often seen as the gold standard of medication ascertainment ${ }^{2}$, but is particularly resource intensive.

A number of studies have assessed the validity and accuracy of self-reported medication data compared with pharmaceutical claims data. Although they have demonstrated that self-reporting is generally appropriate for measuring prescription medication exposure ${ }^{3-6}$, most have evaluated specific medications, or chronically used medications such as cardiovascular medications. ${ }^{4}$ It is possible that the validity of self-reported data varies according to the medication type in terms of clinical indication. For example, there may be issues with capturing intermittent medication use or medications associated with stigma.

Thus, the aim of this study was to compare the accuracy of self-reported prescription-only medication use across a very wide range of medication types with the gold standard method of linking routine data on claims for dispensed prescription-only medications. We used data from a sample of participants from the 45 and Up Study, a large-scale Australian cohort study.

\section{Methods}

\section{Study population}

This study used baseline questionnaire data from the Sax Institute's 45 and Up Study, which includes 267153 men and women aged $\geq 45$ years from New South Wales, Australia, randomly sampled from the Medicare Australia database. ${ }^{7}$ Participants completed a self-administered postal questionnaire. Recruitment was conducted from
February 2006 to April 2009, with an 18\% response rate. For this study, we selected a random sample of 500 individuals who were healthcare concession cardholders, and hence had complete and consistent capture of their dispensed prescriptions through the Pharmaceutical Benefits Scheme (PBS). The sample was enriched for medication use, with $95 \%$ of participants having any self-reported medication recorded as free text, in addition to medications listed in check boxes, described in detail elsewhere. ${ }^{8}$ This study was approved by the UNSW Human Research Ethics Committee, Sydney, Australia (05035).

\section{Self-reported medication data}

In the 45 and Up Study, participants are asked to provide information on medication use in response to the question, "Have you taken any medications, vitamins or supplements for most of the last 4 weeks?" There is a checklist of commonly used medications and a free-text response box for medications not included as checkbox items. The self-reported medicines included both those listed in the check box and those listed in the free-text box. The check-box option included the 32 most common medications, vitamins and supplements used in the Australian population at the time the baseline survey was conducted. The free-text fields were reviewed and coded by medication experts. ${ }^{8}$ If a check-box medication was both ticked and listed in the free-text box, we only counted it once. We defined medication exposure as use of any self-reported prescription medication as listed in the Australian registered product information ${ }^{9}$ in the 4 weeks before the survey (coded as 28 days for the purposes of our analysis). We chose this approach because prescription-only medications are more likely to appear consistently in PBS records. We classified participants as 'unexposed' if there was no record of prescription-only medications in the check box or freetext box.

Medication names were converted to active ingredient names using the Australian registered product information. ${ }^{10}$ Active ingredient names were coded automatically to Anatomical Therapeutic Chemical (ATC) classification codes to chemical substance code level (7 digits), chemical subgroup code level (5 digits), pharmacological subgroup code level (4 digits) or therapeutic subgroup code level (3 digits). The results for the 7-and 3-digit codes are presented in this paper. We did not include vaccines (ATC code J07), because these agents are not typically considered to be medications and they are not used regularly. 
Table 1. Baseline characteristics of the study population $(n=500)$

\begin{tabular}{|c|c|}
\hline Characteristic & Study population \\
\hline Age, mean (SD) years & $70.1(10.3)$ \\
\hline Female, \% (n) & $55.6(278)$ \\
\hline Australia born, \% (n) & $71.0(353)$ \\
\hline Married/de facto ${ }^{a}, \%(n)$ & $60.2(299)$ \\
\hline \multicolumn{2}{|l|}{ Educational qualification, \% (n) } \\
\hline No certificate & $19.5(94)$ \\
\hline School, intermediate certificate/trade & $46.2(223)$ \\
\hline Higher school/leaving certificate & $8.7(42)$ \\
\hline Certificate/diploma & $17.2(83)$ \\
\hline University degree & $8.5(41)$ \\
\hline \multicolumn{2}{|l|}{ Household income, \% (n) } \\
\hline$<\$ 20000$ & $47.4(221)$ \\
\hline$\$ 20000$ to $<\$ 40000$ & $26.6(124)$ \\
\hline$\$ 40000$ to $<\$ 70000$ & $6.4(30)$ \\
\hline$\geq \$ 70000$ & $19.5(91)$ \\
\hline \multicolumn{2}{|c|}{ Alcohol use, number of drinks per week, $\%(n)$} \\
\hline None & $45.5(220)$ \\
\hline $1-14$ & $41.7(202)$ \\
\hline$\geq 15$ & $12.8(62)$ \\
\hline Ever a regular smoker, \% $(n)$ & $47.4(237)$ \\
\hline \multicolumn{2}{|l|}{ Self-rated health, \% ( $n)$} \\
\hline Excellent & $7.7(37)$ \\
\hline Very good & $24.7(119)$ \\
\hline Good & $40.9(197)$ \\
\hline Fair & $22.2(107)$ \\
\hline Poor & $4.6(22)$ \\
\hline Cardiovascular disease $^{\mathrm{b}}, \%(n)$ & $32.8(164)$ \\
\hline $\begin{array}{l}\text { Number of self-reported } \\
\text { prescription-only medicines, mean } \\
\text { (SD) }\end{array}$ & $3.3(2.3)$ \\
\hline
\end{tabular}

$\mathrm{SD}=$ standard deviation

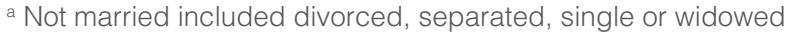

${ }^{b}$ Diabetes, stroke, blood clots or heart disease

Note: Some data are missing for the following characteristics: country of birth $(n=3)$, marital status $(n=3)$, household income $(n=34)$, education $(n=17)$, alcohol use $(n=16)$ and self-rated health $(n=18)$.

\section{Pharmaceutical claims data}

Questionnaire data from participants were linked to PBS data from 2004 to 2011, supplied by the Australian Government Department of Human Services and linked by the Sax Institute. The PBS dataset contains information on subsidised claims for dispensing of prescribed medicines listed on the Schedule of Pharmaceutical Benefits in Australia. During the study period, complete dispensing histories were generally recorded only for healthcare concession cardholders. Hence, the study sample was restricted to these individuals.

We defined distinct medications at the 7-digit ATC level, and therapeutic subgroup using 3-digit codes. We classified 'gold standard' exposure as an individual being dispensed a PBS medicine up to 90 days before the 4-week (28-day) window before the survey, as this dispensing is likely to include the 4-week period relating to the survey response. Hence, an individual was considered 'exposed' to a specific medication if they were dispensed a PBS medicine up to 90 days before the 4-week period before the survey. The 90-day period is well established in the literature and is used for the primary analysis presented in this paper, with 60- and 30day estimates used for sensitivity analysis. ${ }^{11,12}$ The three time periods account for different lengths of time that a dispensed prescription might last in the community.

\section{Data analysis}

The PBS data were considered the gold standard. The main comparisons were performed in relation to the therapeutic subgroup (3-digit ATC) and individual medications (7-digit ATC). In the questionnaire, two check-box medications, namely 'Coversyl (CO9AA04), Coversyl plus (C09BA04)' and 'Avapro (C09DA04), Karvea (C09CA04)' could be assigned two ATC codes because these refer to two different generic names. To account for this, these medications were included for comparison at the 3-digit level only. We calculated sensitivity (i.e. the proportion of PBS medication use that is identified correctly by self-reporting), positive predictive values (PPVs; proportion of self-reported use that is confirmed by the PBS data) and specificity (i.e. proportion of non-use of a PBS medication that is correctly identified by self-reporting) at the 3-and 7-digit ATC levels, and used the 'exact' binomial method to calculate the corresponding 95\% confidence interval $(\mathrm{Cl})$. Data analyses were performed using SAS version 9.3 (SAS Institute, Cary, NC).

\section{Results}

The mean age of participants was 70.1 years (SD 10.3), $55.6 \%$ were female and $71.0 \%$ were born in Australia (Table 1). Compared with the 45 and Up Study cohort members, similar to other concession cardholders, our participants were generally older, were more likely to be born overseas, not be married and have fewer educational qualifications. ${ }^{8}$ Overall, $92.8 \%$ (464) of participants self-reported use of at least one prescriptiononly medication, $6.6 \%(n=33)$ self-reported use of nonprescription medications, and $0.6 \%(n=3)$ did not self-report use of any medication in the previous 4 weeks. 
The proportion of participants with medications recorded and the sensitivity and PPV of self-reported versus PBS data for prescription-only medications for therapeutic medication classes using the 90-day window period are shown in Table 2, and for individual medications in Supplementary Table 1 (available from: hdl.handle.net/1885/119323).

The sensitivity/PPV of self-reported use versus PBS data at the 3-digit ATC level (Table 2) were highest for chronic cardiovascular medications, being 94.4\% (95\% Cl 90.6, 97.0)/96.9\% (95\% Cl 93.7, 98.7), respectively, for lipid-lowering agents; $92.5 \%$ (88.5, 95.4)/97.5\% (94.6, 99.1) for angiotensin agents; 88.8\% (81.2, 94.1)/93.1\% (86.4, 97.2) for beta-blockers; and 88.0\% $(80.3,93.4) / 96.9 \%(91.3,99.4)$ for calcium-channel blockers. The sensitivity of self-reported centrally acting medications was $78.9 \%(95 \% \mathrm{Cl} 69.0,86.8)$ for psychoanaleptics; $42.1 \%(26.3,59.2)$ for analgesics; $26.0 \%(16.5,37.6)$ for psycholeptics; and 4.8\% (2.0, 9.7) for antibacterial agents. Overall, the PPVs for these medication types were $\geq 65 \%$, with PPVs of $\geq 75 \%$ for $79 \%$ of the medications examined.

The sensitivity/PPV of self-reported use versus PBS data at the 7-digit ATC level (see Supplementary Table 1, available from: hdl.handle.net/1885/119323) varied across individual medications. The highest sensitivity/PPV for self-reported versus claims data at the 7-digit ATC level were 96.9\% (95\% Cl 83.8, 99.9)/96.9\% (95\% Cl 83.8, 99.9) for warfarin; $94.5 \%(88.4,98.5) / 92.0 \%(85.3,96.3)$ for atorvastatin; 94.3\% (80.8, 99.3)/84.6\% (69.5, 94.1) for pantoprazole, and 93.3\% (81.7,98.6)/95.5\% (84.5, 99.4) for atenolol. The lowest sensitivities were observed for a range of individual medications, being $16.7 \%$ (95\% Cl 5.6, 34.7) for temazepam; 15.2\% (5.1, 31.9) for perindopril; $11.5 \%(4.4,23.4)$ for irbesartan; $11.1 \%(1.4$, $34.7)$ for oxazepam, and $3.3 \%(0.1,17.2)$ for amoxicillin.

Overall, the PPVs for individual medications were $\geq 75 \%$ for $81 \%$ of the medications examined. Findings from analyses using 30- and 60-day exposure periods (see Supplementary Table 2, available from: hdl.handle.net/1885/119323) did not differ from those in the main analyses.

Data on specificity using the 90-day period are provided in Supplementary Table 3 (available from: hdl.handle.net/1885/119323). Specificities of self-reported use versus PBS data at the 3-digit ATC level ranged from $96.5 \%(95 \%$ Cl 94.4, 97.9) for cardiac therapy agents to $100 \%$ for ophthalmologic agents.

\section{Discussion}

The findings of this study indicate that sensitivity and PPVs for self-reported versus PBS claims data for prescription-only medications are good overall, and vary by medication type. The sensitivity and PPVs for self-reported cardiovascular medications were good to excellent compared with the claims data. This is consistent with previous studies, which have shown that self-reported data can be used to accurately identify the use of statins and diuretics. . $13^{3}$

Our results are similar to a recently published paper that compared a self-administered mailed form for specific medications, and found near-perfect agreement between self-reported data and pharmacy records for recent use of long-term medicines such as statins, betablockers and calcium-channel blockers. ${ }^{14}$ In this study, participants were asked to refer to their prescription labels while completing the form; therefore, this approach may be useful to collect self-reported data on recent medication use. The accuracy of self-reporting exposure to cardiovascular medications is consistently good across studies, regardless of study population, study designs and methods used to capture medication use. ${ }^{13-15}$

Our study found that self-reported data of the type used here may be less useful for identifying exposure to medications such as anxiolytics, sedatives, analgesics and antibacterial agents. These medications are typically taken for short periods or intermittently, so may be less likely to be identified as having been used for "most of the last 4 weeks" by study participants. In general, the findings for sensitivities and PPVs for medication classes (3-digit level) reflected consistent findings for individual medications (7-digit level), including for lipidmodifying agents, medications for acid-related disorders, beta-blockers, antithrombotic agents, diuretics and calcium-channel blockers. However, findings could vary within medication classes, as demonstrated by high sensitivities for most angiotensin II-receptor antagonists and angiotensin-converting enzyme inhibitors, apart from irbesartan and perindopril. The reasons for this variation are unclear, but should be borne in mind in future studies.

Importantly, PPVs remained relatively high for most of the medication types, including those with low prevalence. This means that, for exposure classification, most of those identified as exposed to the medicine by self-report will have been exposed. Even if sensitivity is not particularly high, sound comparisons can be made between exposed and unexposed when medication exposure is relatively uncommon. Contamination of the unexposed group (i.e. low sensitivity/inclusion of people exposed to a medication in the unexposed or control group) may occur where medication exposure is common, and would generally mean that estimates of relative risk would be biased towards the null (i.e. towards a relative risk of 1), leading to more conservative findings. However, low sensitivity and specificity may compromise study power, and complementary information on sensitivity and specificity, clinical context, research design, and research questions should be considered. Hence, self-reported data are likely to be useful to identify and compare exposed individuals for many medications.

To our knowledge, our study is the first to compare the accuracy of self-reported medication data with pharmaceutical claims data for a range of medications. This is essential to better understand the merits of self- 
Table 2. Frequency, sensitivity and positive predictive value of 45 and Up Study self-reported data for prescription-only medications to 3-digit level ATC classification compared with PBS pharmaceutical claims data (gold standard) considering 90-day exposure definition

\begin{tabular}{|c|c|c|c|c|c|}
\hline \multirow[b]{2}{*}{ Therapeutic group (ATC class) ${ }^{a}$} & \multicolumn{3}{|c|}{$\begin{array}{l}\text { Participants identified with specified } \\
\text { medications }\end{array}$} & \multirow[b]{2}{*}{$\begin{array}{l}\text { Sensitivity } \\
\%(95 \% \mathrm{Cl})\end{array}$} & \multirow[b]{2}{*}{$\begin{array}{c}\text { PPV } \\
\%(95 \% \mathrm{Cl})\end{array}$} \\
\hline & $\begin{array}{l}\text { PBS entry } \\
\%(n)\end{array}$ & $\begin{array}{c}\text { Survey } \\
\text { entry } \\
\%(n)\end{array}$ & $\begin{array}{l}\text { Correct }^{b} \\
\text { survey } \\
\text { entry } \\
\%(n)\end{array}$ & & \\
\hline $\begin{array}{l}\text { Agents acting on the renin-angiotensin system } \\
\text { (C09) }\end{array}$ & $50.4(252)$ & $47.8(239)$ & $46.6(233)$ & $92.5(88.5,95.4)$ & $97.5(94.6,99.1)$ \\
\hline Lipid-modifying agents (C10) & $46.2(231)$ & $45.0(225)$ & $43.6(218)$ & $94.4(90.6,97.0)$ & $96.9(93.7,98.7)$ \\
\hline Drugs for acid-related disorders (A02) & $36.0(180)$ & $33.0(165)$ & $31.0(155)$ & $86.1(80.2,90.8)$ & $94.0(89.1,97.1)$ \\
\hline Antibacterial agents for systemic use (J01) & $29.0(145)$ & $1.8(9)$ & $1.4(7)$ & $4.8(2.0,9.7)$ & $77.9(40.0,97.2)$ \\
\hline Calcium-channel blockers (C08) & $21.6(108)$ & $19.6(98)$ & $19.0(95)$ & $88.0(80.3,93.4)$ & $96.9(91.3,99.4)$ \\
\hline Beta-blocking agents (C07) & $21.4(107)$ & $20.4(102)$ & $19.0(95)$ & $88.8(81.2,94.1)$ & $93.1(86.4,97.2)$ \\
\hline Psychoanaleptic agents (N06) & $18.0(90)$ & $15.2(76)$ & $14.2(71)$ & $78.9(69.0,86.8)$ & $93.4(85.3,97.8)$ \\
\hline Antithrombotic agents (B01) & $15.0(75)$ & $11.8(59)$ & $11.6(58)$ & $77.3(66.2,86.2)$ & $98.3(90.9,100.0)$ \\
\hline Psycholeptics (N05) & $14.6(73)$ & $5.8(29)$ & $3.8(19)$ & $26.0(16.5,37.6)$ & $65.5(45.7,82.1)$ \\
\hline Obstructive airway disease agents (R03) & $14.2(71)$ & $5.2(26)$ & $4.2(21)$ & $29.6(19.3,41.6)$ & $80.7(60.7,93.5)$ \\
\hline Diuretics (C03) & $13.4(67)$ & $15.0(75)$ & $12.0(60)$ & $89.6(79.7,95.7)$ & $80.0(69.2,88.4)$ \\
\hline Anti-inflammatory and antirheumatic agents (M01) & $12.0(60)$ & $6.8(34)$ & $5.8(29)$ & $48.3(35.2,61.6)$ & $85.3(68.9,95.1)$ \\
\hline Diabetes agents (A10) & $11.6(58)$ & $10.6(53)$ & $10.4(52)$ & $89.7(78.8,96.1)$ & $98.1(89.9,100.0)$ \\
\hline Ophthalmologic agents (S01) & $9.4(47)$ & $3.6(18)$ & $3.6(18)$ & $38.3(24.5,53.6)$ & 100 \\
\hline Bone disease agents (M05) & $9.2(46)$ & $5.8(29)$ & $5.0(25)$ & $54.4(39.0,69.1)$ & $86.2(68.3,96.1)$ \\
\hline Sex hormones and modulators (G03) & $9.0(45)$ & $6.0(30)$ & $3.8(19)$ & $42.2(27.7,57.9)$ & $63.3(43.9,80.1)$ \\
\hline Corticosteroids, dermatological agents (D07) & $8.4(42)$ & $0(0)$ & $0(0)$ & 0 & na \\
\hline Cardiac therapy agents (C01) & $8.4(42)$ & $9.8(49)$ & $6.6(33)$ & $78.6(63.2,89.7)$ & $67.4(52.5,80.1)$ \\
\hline Anti-gout agents (M04) & $8.2(41)$ & $8.0(40)$ & $7.0(35)$ & $85.4(70.8,94.4)$ & $87.5(73.2,95.8)$ \\
\hline Analgesics (N02) & $7.6(38)$ & $4.6(23)$ & $3.2(16)$ & $42.1(26.3,59.2)$ & $69.5(47.1,86.8)$ \\
\hline Corticosteroids for systemic use (H02) & $7.0(35)$ & $4.4(22)$ & $3.8(19)$ & $54.3(36.7,71.2)$ & $86.3(65.1,97.1)$ \\
\hline Thyroid agents (H03) & $5.8(29)$ & $7.8(39)$ & $4.6(23)$ & $79.3(60.3,92.0)$ & $59.0(42.1,74.4)$ \\
\hline Otologicals, anti-infectives (S02) & $4.2(21)$ & $0(0)$ & $0(0)$ & 0 & na \\
\hline Antiepileptics (N03) & $4.0(20)$ & $4.0(20)$ & $3.0(15)$ & $75.0(50.9,91.3)$ & $75.0(50.9,91.3)$ \\
\hline Antihypertensives (C02) & $3.4(17)$ & $3.0(15)$ & $2.4(12)$ & $70.6(44.0,89.7)$ & $80.0(51.9,95.7)$ \\
\hline $\begin{array}{l}\text { Antidiarrheals, intestinal anti-inflammatory/anti- } \\
\text { infective agents (A07) }\end{array}$ & $2.4(12)$ & $1.0(5)$ & $0.8(4)$ & $33.3(9.9,65.1)$ & $80.4(28.4,99.5)$ \\
\hline
\end{tabular}

ATC = Anatomical Therapeutic Chemical; na = not applicable; PBS = Pharmaceutical Benefits Scheme; PPV = positive predictive value

a Table sorted by order of pharmaceutical claims data frequency

${ }^{b}$ Correct survey entry is where a pharmaceutical claims record exists for the same medication

Note: Only medication classes with 10 or more entries identified using pharmaceutical claims data are presented. 
reported data across the full spectrum of prescribed medications. Additional analyses were conducted to account for different exposure periods, because the degree of misclassification of medication exposure may vary across different fixed-time periods and may partly explain differences observed across studies. The PBS dataset was our gold standard. However, this is imperfect because participants may be dispensed medications that they do not use, and pharmaceutical claims datasets in Australia do not capture all prescription medications, including medications supplied by hospitals or available as private prescriptions. These two factors may oppose each other, meaning that PBS dispensings may overestimate exposure to medications that were not then used (this might apply more to short-term or oneoff dispensings) and may underestimate exposure to certain medicines. Hence, some of the PBS dispensings that were not accompanied by self-reporting may be medications that were not taken, consistent with the finding of low validity for use of certain medications that are generally taken short term or as a one-off, and some of the 'false positives' observed in our study may be medications that participants were receiving from other sources. These factors suggest that our findings regarding validity may be underestimated.

To allow reliable comparisons between selfreporting and dispensing data, our study was restricted to concession cardholders. This group is generally older and more likely to have chronic disease and be prescribed multiple medications than the general population. These factors may decrease the accuracy of self-reporting, indicating the potential for the validity of self-reporting to be somewhat better in the general population. We enriched the sample for medication users - about $99 \%$ of our sample reported use of any medication during the past 4 weeks, compared with $91 \%$ of all healthcare concession cardholders in the broader 45 and Up Study. This is unlikely to have had a large effect on validity of reporting for medication classes and individual medications, but a slight elevation in the tendency to self-report cannot be excluded. The survey structure may have also affected the accuracy of self-reported medications. ${ }^{16}$ One study found that some medications, such as antibacterial agents, are more likely to be reported if participants are prompted (73\% sensitivity) rather than asked to list medications in open-ended question (50\% sensitivity). ${ }^{17}$ Interestingly, although studies have noted that participants may be less inclined to report the use of psychoanaleptics such as antidepressants due to self-stigmatisation ${ }^{18}$, this was not observed in our study.

\section{Conclusion}

In summary, self-reported medication data of the type examined here are likely to be useful for identifying exposure to a range of prescription medications, particularly those used long term, in future pharmacoepidemiological studies. However, caution is warranted when using such data to identify prescription-only medications used in the short term or intermittently. These findings have important implications for design and interpretation of pharmacoepidemiological studies using self-reported data to examine use and safety of prescription medications in real-world settings.

\section{Acknowledgements}

This research was completed using data collected through the 45 and Up Study (www.saxinstitute.org.au/ our-work/45-up-study). The 45 and Up Study is managed by the Sax Institute in collaboration with major partner Cancer Council NSW, and other partners: the National Heart Foundation of Australia (NSW Division), the NSW Ministry of Health, beyondblue, the NSW Government Family and Community Services - Carers, Ageing and Disability Inclusion, and the Australian Red Cross Blood Service. We thank the many thousands of people participating in the 45 and Up Study. This study was funded by the National Health and Medical Research Council of Australia (NHMRC project grant number 1024450 and NHMRC Centre of Research Excellence in Medicines and Ageing, 1060407). DG is supported by an NHMRC Early Career Fellowship and EB is supported by an NHMRC Senior Research Fellowship.

\section{Competing interests}

None declared

\section{Author contributions}

DG and WD contributed equally to the article, including its conception, design, data analysis, drafting and editing. $\mathrm{SP}, \mathrm{SH}$ and EB contributed to the conception, design, drafting and editing of the article. All authors made an intellectual contribution.

\section{References}

1. Page E, Kemp-Casey A, Korda R, Banks E. Using Australian Pharmaceutical Benefits Scheme data for pharmacoepidemiological research: challenges and approaches. Public Health Res Pract. 2015;25(4):e2541546

2. Lau HS, de Boer A, Beuning KS, Porsius A. Validation of pharmacy records in drug exposure assessment. J Clin Epidemiol. 1997;50(5):619-25.

3. Klungel $\mathrm{OH}$, de Boer $\mathrm{A}$, Paes $\mathrm{AH}$, Herings RM, Seidell JC, Bakker A. Agreement between self-reported antihypertensive drug use and pharmacy records in a population-based study in The Netherlands. Pharm World Sci. 1999;21(5):217-20. 
4. Pit SW, Byles JE, Cockburn J. Accuracy of telephone self-report of drug use in older people and agreement with pharmaceutical claims data. Drugs Aging. 2008;25(1):71-80.

5. Nielsen MW, Sondergaard B, Kjoller M, Hansen EH. Agreement between self-reported data on medicine use and prescription records vary according to method of analysis and therapeutic group. J Clin Epidemiol. 2008;61(9):919-24.

6. Banks E, Beral V, Cameron R, Hogg A, Langley N, Barnes I, et al. Agreement between general practice prescription data and self-reported use of hormone replacement therapy and treatment for various illnesses. J Epidemiol Biostat. 2001;6(4):357-63.

7. 45 and Up Study Collaborators, Banks E, Redman S, Jorm L, Armstrong B, Bauman A, et al. Cohort profile: the 45 and up study. Int J Epidemiol. 2008;37(5):941-7.

8. Gnjidic D, Pearson SA, Hilmer SN, Basilakis J, Schaffer AL, Blyth FM, Banks E; High Risk Prescribing Investigators. Manual versus automated coding of free-text self-reported medication data in the 45 and Up Study: a validation study. Public Health Res Pract. 2015;25(2):e2521518.

9. Donohoo E, Editor. MIMS Annual. Sydney: CMPMedica; 2015

10. WHO Collaborating Centre for Drug Statistics Methodology. The anatomical therapeutic chemical classification system. Oslo: WHO Collaborating Centre for Drug Statistics Methodology, Norwegian Institute of Public Health; 2015. Available from: www.whocc.no/atcddd

11. Hallas J, Dall M, Andries A, Andersen BS, Aalykke C, Hansen JM, et al. Use of single and combined antithrombotic therapy and risk of serious upper gastrointestinal bleeding: population based case-control study. BMJ. 2006;333(7571):726.
12. King MA, Purdie DM, Roberts MS. Matching prescription claims with medication data for nursing home residents: implications for prescriber feedback, drug utilisation studies and selection of prescription claims database. J Clin Epidemiol. 2001;54(2):202-9.

13. Smith NL, Psaty BM, Heckbert SR, Tracy RP, Cornell ES. The reliability of medication inventory methods compared to serum levels of cardiovascular drugs in the elderly. J Clin Epidemiol. 1999;52(2):143-6.

14. Drieling RL, LaCroix AZ, Beresford SA, Boudreau DM, Kooperberg C, Heckbert SR. Validity of self-reported medication use compared with pharmacy records in a cohort of older women: findings from the Women's Health Initiative. Am J Epidemiol. 2016;184(3):233-8.

15. Sjahid SI, van der Linden PD, Stricker BH. Agreement between the pharmacy medication history and patient interview for cardiovascular drugs: the Rotterdam elderly study. Br J Clin Pharmacol. 1998;45(6):591-5.

16. Gama H, Correia S, Lunet N. Questionnaire design and the recall of pharmacological treatments: a systematic review. Pharmacoepidemiol Drug Saf. 2009;18(3):175-87.

17. Metlay JP, Hardy C, Strom BL. Agreement between patient self-report and a Veterans Affairs national pharmacy database for identifying recent exposures to antibiotics. Pharmacoepidemiol Drug Saf. 2003;12(1):9-15.

18. Knudsen P, Hansen EH, Traulsen JM. Perceptions of young women using SSRI antidepressants a reclassification of stigma. Int J Pharm Pract. 2002; 10:243-52.

\section{Copyright: (c)}

(C) 2017 Gnjidic et al. This article is licensed under the Creative Commons Attribution-NonCommercial-ShareAlike 4.0 International Licence, which allows others to redistribute, adapt and share this work non-commercially provided they attribute the work and any adapted version of it is distributed under the same Creative Commons licence terms. See: www.creativecommons.org/licenses/by-nc-sa/4.0/ 